Annuaire suisse de politique de développement

$17 \mid 1998$

Propriété intellectuelle : quels enjeux pour les pays en développement ?

\title{
7. Culture et science
}

\section{(2) OpenEdition}

\section{Journals}

Édition électronique

URL : http://journals.openedition.org/aspd/772

DOI : $10.4000 /$ aspd. 772

ISSN : 1663-9669

Éditeur

Institut de hautes études internationales et du développement

Édition imprimée

Date de publication : 1 avril 1998

Pagination : 263-272

ISSN : 1660-5934

Référence électronique

«7. Culture et science», Annuaire suisse de politique de développement [En ligne], 17 | 1998, mis en ligne le 31 juillet 2012, consulté le 08 septembre 2020. URL : http://journals.openedition.org/aspd/772

DOI : https://doi.org/10.4000/aspd.772 


\section{CULTURE ET SCIENCE}

\section{1. ÉDUCATION AU DÉVELOPPEMENT}

La globalisation qui s'est produite ces dernières années dans de nombreux domaines, tels que le commerce, la finance, les communications, la culture, la politique, les conséquences environnementales de certains modes de vie, les migrations, a eu pour conséquence une profonde déstabilisation de l'individu. Cette déstabilisation est provoquée par la disparition de modèles familiers au «profit» d'une homogénéisation culturelle, par la complexité croissante des relations internationales, par des conséquences à l'échelon planétaire de problèmes de pauvreté ou d'environnement qui rendent plus difficile la mise en place de solutions. Parents, enseignants et éducateurs, confrontés aux questions et interrogations des jeunes face à une vision reflétant une inquiétude face au monde et à l'avenir, doivent gérer leurs propres incertitudes et apporter des éléments de réponse aux sollicitations des enfants.

Parallèlement la globalisation a provoqué, notamment à la Direction du développement et de la coopération (DDC) et auprès des organisations non gouvernementales, une prise de conscience qu'une interdépendance mondiale grandissante implique un engagement pédagogique afin de préparer les jeunes au monde de demain. Dès 1970, la DDC s'est mise à publier des outils pédagogiques ayant pour but de mettre en évidence les causes du sous-développement. Des organisations privées, telles que la Communauté de travail, qui créa en 1975 la Schulstelle Dritte Welt de Berne, en 1981 le Service école Tiers Monde à Lausanne et en 1987 le Centro Terzo Mondo à Lugano, ou encore le Comité suisse pour l'UNICEF, la Déclaration de Berne notamment, plaçaient au centre de leur travail pédagogique la question des possibilités d'influence de l'individu. Créé en 1982, le Forum «Ecole pour un seul monde», regroupait la plupart des organisations engagées pour une sensibilisation des jeunes aux domaines des relations Nord-Sud, des droits de l'homme, de l'environnement, de la lutte contre le racisme et de la pédagogie interculturelle.

En 1987, les organisations membres du Forum «Ecole pour un seul monde» ont tenté de définir le concept de l'éducation au développement «comme un encouragement à l'épanouissement personnel des enfants et des jeunes au sein de leur communauté, locale et globale. Dans ce processus les enfants apprennent à se considérer toujours plus comme les membres d'un monde en transformation dans lequel tous les humains ont besoin les uns des autres. L'éducation au développement nous encourage à prendre une part active à l'élaboration de l'avenir et nous engage à défendre des valeurs fondamentales telles que la dignité, la justice et la solidarité dans la communauté mondiale»'.

1. Rapport de la Commission pédagogique du Forum «Ecole pour un seul monde», «Réflexions sur l'éducation dans un monde interdépendant», version inédite en français, septembre 1996, p. 8. 


\section{$\square$ Création de la fondation Education et Développement}

Février 1997 marque un pas très important, en Suisse, dans la prise en considération de la nécessité de renforcer l'éducation au développement. Différents acteurs, la Conférence intercantonale de l'instruction publique (CIIP), la Direction du développement et de la coopération (DDC), certaines organisations non gouvernementales membres du Forum «Ecole pour un seul monde», ainsi que les associations d'enseignants, ont créé ensemble la fondation Education et Développement. Lors de la conférence de presse, Walter Fust, directeur de la DDC, a souligné que «s'il est vrai que la DDC soutient financièrement depuis près de vingt ans le travail pédagogique des organisations d'entraide privées, on peut constater que l'intensification de la collaboration avec les associations d'enseignants et les cantons - souverains en matière d'instruction publique - constitue un pas important et indispensable pour que l'éducation de la jeunesse à une vision globale du monde bénéficie d'un large appui».

La création de cette fondation est le fruit d'un long processus. En 1993, les membres du Forum «Ecole pour un seul monde» ont décidé d'analyser l'offre des ONG (leur travail en matière d'éducation au développement, la production d'outils pédagogiques et des activités proposées, etc.) et l'ont confrontée aux besoins des enseignants. Les conclusions de cette enquête, publiée sous le titre L'apprentissage global en Suisse - Analyse de la situation, des attentes et des perspectives $^{2}$, faisaient apparaître la nécessité d'une meilleure coordination du travail des ONG afin de faciliter aux enseignants l'introduction dans leur enseignement des thèmes relatifs à l'éducation au développement. D'autre part, l'Agenda 21, le plan d'action du Sommet de la Terre de Rio, demandait en 1992 à tous les pays d'intensifier leurs efforts pour que les questions d'environnement et de développement s'implantent véritablement dans l'éducation.

La mise en place de la fondation s'est faite progressivement durant l'année 1997, les activités des services école de la Communauté de travail et du Forum «Ecole pour un seul monde» étant transférées à la nouvelle fondation, avant la dissolution de ces organes. La fondation Education et Développement est tout d'abord un interlocuteur pour les enseignants, les centres de documentation pédagogique, les responsables du perfectionnement des enseignants, les membres des commissions des plans d'étude. Elle identifie les besoins auprès des enseignants, évalue les outils pédagogiques et en favorise la diffusion, encourage l'innovation à l'école et dans les plans d'étude, participe à la formation initiale des enseignants et à leur perfectionnement.

La fondation a pour but d'encourager une éducation qui transmet des savoirs et favorise la perception des rapports globaux, qui présente des possibilités concrètes d'action et qui développe la capacité de l'individu à établir des échanges par-delà les frontières linguistiques, culturelles et géographiques. A moyen terme, la fondation Education et Développement soutiendra financièrement des outils pédagogiques et des projets éducatifs destinés aux écoles et, dans un deuxième temps, à la formation hors milieu scolaire.

2. Forum «Ecole pour un seul monde», L'apprentissage global en Suisse - Analyse de la situation, des attentes et des perspectives, 1995. 
La fondation assure ses services à partir de quatre emplacements: le service central de Berne ${ }^{3}$ assume des tâches à l'échelon national et international, et trois services régionaux (Zurich, Lausanne et Lugano) se chargent du service conseil et de l'appui aux écoles et au corps enseignant de tous les degrés, ainsi que de l'évaluation des moyens d'enseignement et de la formation initiale et continue.

\section{$\square$ Enquête sur la vision du monde chez les jeunes}

A l'initiative du Forum «Ecole pour un seul monde» et de la DDC, une vaste enquête a été menée en 1996 par l'Institut de pédagogie de l'Université de Berne, ayant pour objet la vision du monde chez les jeunes. Le rapport final, «La Suisse, les pays en développement et l'interdépendance mondiale vus par les jeunes», a été publié en juin $1997^{4}$. Cette étude donne suite à une enquête similaire réalisée en $1985^{5}$ et permet de vérifier si l'attitude et la vision des jeunes ont changé au cours de ces dix dernières années.

L'enquête a quatre principaux domaines thématiques: la coxistence, en Suisse, de jeunes de nationalité différente, l'image que les jeunes ont de la Suisse, celle qu'ils ont des pays en développement ainsi que leur perception de l'interdépendance mondiale. Le questionnaire se rapportait aux connaissances des jeunes, à leur attitude, à leur comportement et à leur disposition à agir dans les quatre domaines cités. A partir d'un échantillon représentatif, près de 5000 élèves âgés de 13 à 16 ans des trois régions linguistiques ont participé à l'enquête. Un questionnaire très complet de 88 questions leur a été soumis, dont les principaux éléments de réponse sont présentés ci-dessous 6 .

\section{- Image de la Suisse}

Afin de percevoir l'image que les jeunes ont de la Suisse, un certain nombre de problèmes leur étaient présentés. C'est la drogue qui constitue le problème majeur, suivie du chômage et du sida. Un deuxième ensemble de préoccupations concerne la violence, la destruction de l'environnement et le racisme, considérés comme relativement importants. Les réfugiés, l'Europe, l'énergie nucléaire, la pauvreté et la surpopulation, bien qu'importants, apparaissent ensuite. La situation politique, la malnutrition, la formation et l'accès aux soins constituent pour leur part des problèmes mineurs ou inexistants'. La comparaison avec l'enquête de 1985 laisse apparaître que l'environnement, figurant à la première place il y a une dizaine d'années, a été «relégué» à la cinquième position lors de la récente enquête.

3 Fondation Education et Développement, secrétariat suisse, Monbijoustrasse 31, case postale 8366, 3001 Berne, tél. 031/382.80.80, fax 031/382.80.82.

4. «La Suisse, les pays en développement et l'interdépendance mondiale vus par les jeunes», Institut de pédagogie de l'Université de Berne et Forum «Ecole pour un seul monde», Berne, juin 1997. Disponible en allemand uniquement, environ 500 pages. Un abrégé de l'étude est disponible en français et en allemand: W. Herzog, J. Guldimann, T. Oergerli, La Suisse, les pays en développement et l'interdépendance mondiale vus par les jeunes, enquête représentative menée en Suisse auprès des élèves de 13 à 16 ans, Institut de pédagogie de l'Université de Berne et Forum «Ecole pour un seul monde», Berne, juin 1997.

5. Un résumé de cette enquête a été plublié dans L'Educateur, «Enquête - Le monde dans lequel nous vivons», mai 1986.

6. D'autres questions relatives à la coopération au développement, les amitiés entre Suisses et étrangers, les motifs justifiant l'asile, la disposition des jeunes à s'engager en faveur des PVD, etc., sont largement présentées dans le rapport final.

7. Les résultats présentés sont les résultats globaux des jeunes; des différences peuvent apparaître lors d'une analyse plus fine, selon la région linguistique, le sexe ou l'âge des jeunes interrogés. Se référer à l'étude complète pour de plus amples détails. 


\section{- Perception des pays en développement}

L'image que les jeunes se font des pays en développement est marquée par des aspects négatifs: la réalité de ces pays se caractérise en premier lieu par la pauvreté, la faim, la surpopulation et la maladie. En dix ans, la perception que les jeunes s'en font n'a pratiquement pas changé. Selon eux, les principaux problèmes des PVD sont la sous-alimentation, la pauvreté, le chômage, la guerre et l'accès insuffisant aux soins médicaux.

\section{- Relation entre la Suisse et les pays en développement}

De l'avis des jeunes, les facteurs responsables de la situation des pays en développement sont les grandes entreprises multinationales, le climat défavorable, des structures sociales injustes, un nombre d'enfants élevé et les taux d'intérêt élevés des banques (problème de la dette). De l'avis des élèves, les difficultés des pays en développement ne doivent pas être mises sur le compte de la «vente de marchandises de mauvaise qualité» et de la "paresse».

\section{- Coexistence en Suisse de personnes de nationalité différente}

Pour cerner l'attitude des jeunes face à la population étrangère, trois propositions de coexistence leur étaient soumises: la tolérance (les étrangers devraient pouvoir vivre en Suisse en accord avec les habitudes qu'ils avaient dans leur pays d'origine), la délimitation (les étrangers devraient, par leurs habitudes, ne pas déranger les habitudes des Suisses) et l'adaptation (les étrangers en Suisse devraient conformer leurs habitudes à celles des Suisses). La moitié des jeunes $(48,9 \%)$ s'exprime en faveur de la deuxième variante. Les autres choisissent l'adaptation $(28,8 \%)$ plutôt qu'une coxistence fondée sur la tolérance $(22,3 \%)$.

\section{- Sources d'information}

L'enquête cherchait également à savoir d'où les jeunes détenaient leurs connaissances relatives aux pays en développement. La télévision, citée par $85 \%$ des jeunes, s'avère la source d'information la plus importante, suivie par l'école $(68 \%)$ et les journaux $(66 \%)$. La famille joue à cet égard un rôle moins important, mais elle est citée tout de même par une bonne moitié des jeunes $(53 \%)$. La comparaison avec l'enquête de 1985 révèle que l'école est passée du troisième au second rang; par ailleurs les élèves étaient en 1996 une fois et demie plus nombreux qu'en 1985 à la citer comme source d'information.

L'image que les jeunes se font des PVD n'a pratiquement pas changé au cours des dix dernières années. Elle est stéréotypée et correspond, dans une large mesure, à l'image diffusée par les émissions de télévision. Cependant, l'école, comme source d'information, est passée en deuxième position. Les résultats de cette enquête vont être particulièrement précieux pour concevoir au mieux l'offre pédagogique.

\section{SOURCES}

Education au développement, B. Riondet, Paris, Hachette Education, 1995.

Dossier de presse du 18 février 1997, «Lancement de la fondation Education et Développement », disponible auprès du service de presse de la DDC, tél. 031/322.34.88.

Rapport de la Commission pédagogique du Forum «Ecole pour un seul monde», «Réflexions sur l'éducation dans un monde interdépendant», version inédite en français, septembre 1996.

W. Herzog, J. Guldimann, T. Oergerli, La Suisse, les pays en développement et l'interdépendance mondiale vus par les jeunes, enquête représentative menée en Suisse auprès des élèves de 13 à 16 ans, abrégé de l'étude «La Suisse, les pays en développement et l'interdépendance mondiale vus par les jeunes», Institut de pédagogie de l'Université de Berne et Forum «Ecole pour un seul monde», Berne, juin 1997, 60 pages.

Mosquito, «Neue Strukturen für Bildung und Entwicklung», Nr. 7/97. 


\subsection{RECHERCHE ET COOPÉRATION SCIENTIFIQUE}

\section{$\square$ La Suisse dans un monde en mutation - Conclusions du PNR 28}

Le Programme national de recherche 28 (PNR 28), «Economie extérieure et politique de développement», s'est terminé en septembre 1997 avec la publication du rapport final rédigé par Rolf Kappel et Olivier Landmann ${ }^{8}$. Les 26 projets de recherche menés de 1989 à 1996 étaient axés sur les thèmes de l'économie extérieure et de la politique du développement. Le rapport final a été présenté lors d'un séminaire public à Berne, le 25 septembre 1997. Plusieurs rapports de synthèse abordent la politique extérieure de la Suisse envers les pays en développement (voir encadré plus loin). Parmi les résultats essentiels des projets en lien avec la politique de la Suisse envers les pays en développement, on peut relever les conclusions ou propositions suivantes.

- La globalisation n'est pas responsable des problèmes rencontrés par l'économie suisse. Si la place économique suisse connaît des difficultés, c'est une conséquence non pas de délocalisations, mais d'un secteur national surprotégé. Un fossé existe entre un secteur international compétitif à l'étranger (sous la pression de la concurrence) et des surcapacités internes (dans le secteur des banques par exemple).

- La politique suisse à l'égard des travailleurs étrangers freine la croissance économique. La réglementation sur l'immigration a été détournée pour appuyer des branches économiques structurellement faibles, orientées uniquement sur le marché intérieur (secteur de la construction par exemple). L'auteur de l'une des recherches, Thomas Straubhaar, propose une politique des migrations plus orientée sur les besoins précis du marché suisse en travailleurs qualifiés. Proposition novatrice: d'une part tous les citoyens du monde pourraient avoir en principe le droit d'immigrer en Suisse, pour autant qu'ils payent une taxe. La taxe devrait selon lui indemniser la Suisse pour l'utilisation des infrastructures et prestations publiques, ainsi que pour couvrir les frais d'intégration et d'assimilation. La politique extérieure de la Suisse devrait d'autre part contribuer à l'élimination des causes des migrations, pour que les migrants potentiels puissent trouver dans leurs pays d'origine les conditions les incitant à rester chez eux.

- Les entreprises suisses (et la diplomatie) n'ont pas encore suffisamment saisi leurs chances sur les marchés les plus dynamiques en Asie.

- La Suisse se trouve face au même défi que les pays en développement: ouvrir son économie au monde. Le repli sur soi et le frein aux ajustements structurels ont un énorme coût pour l'économie.

- Selon les auteurs de certains projets de recherche du PNR 28, il n'existe à ce jour aucune alternative convaincante aux programmes d'ajustement structurel appliqués dans les pays en développement. Ces programmes sont souvent critiqués en raison de leurs effets néfastes sur les couches de population les plus pauvres, notamment par les suites d'une diminution des dépenses sociales des gouvernements (éducation, santé). Il est cependant, selon des

8. Rolf Kappel/Olivier Landmann, La Suisse dans un monde en mutation - Economie extérieure et politique du développement: défis et perspectives, Fribourg, Editions Universitaires Fribourg, 1997, 456 pages. 
études, difficile d'évaluer les impacts sociaux négatifs de l'ajustement sur les plus pauvres (difficile d'isoler l'effet du programme d'ajustement des conséquences d'une économie en crise). Les critiques ne tiennent souvent pas compte de la manière dont la situation sociale aurait évolué sans ajustement. Les auteurs vont même plus loin en affirmant que l'on ne peut pas exiger des programmes d'ajustement structurel de résoudre plusieurs problèmes à la fois: stabiliser l'économie d'un pays et l'ouvrir à l'extérieur, lutter contre la pauvreté et en même temps favoriser le développement durable. Un seul train de mesures ne peut pas permettre d'atteindre ces quatre objectifs à la fois.

\section{QUELQUES PROJETS DU PNR 28 SUR LA POLITIQUE DE DÉVELOPPEMENT}

- Gilbert Etienne/Jean-Luc Maurer/Philippe Regnier/Christine Renaudin, Suisse-Asie - Pour un nouveau partenariat Inde - Pakistan - Chine - Indonésie.

L'économie privée suisse s'est bien implantée en Asie et les échanges commerciaux se sont développés. Mais l'engagement des pouvoirs publics et des entreprises pourrait être beaucoup plus poussé (diplomatie commerciale, formation des cadres).

- Jakob Juchler, Krise und Umbruch in Osteuropa.

Vue d'ensemble du processus de transformation en Europe de l'Est. Le meilleur moyen de soutenir la démocratie et l'économie de marché est l'ouverture des frontières aux produits venant de l'Est.

口 Thomas Straubhaar, Von der Ausländer- zur Migrationspolitik.

L'auteur plaide pour une nouvelle politique d'immigration: soumettre l'immigration à des règles claires (politique intérieure) et éliminer les causes de l'immigration (politique extérieure).

- Joseph Hunkeler, L'impossibilité de rembourser la dette.

Etude du programme d'ajustement structurel au Burundi. La libéralisation précipitée du commerce extérieur et le désendettement interne de l'Etat ont été à l'origine d'un grave surendettement extérieur.

- Jean-Max Baumer, Le programme d'ajustement structurel en Bolivie.

Analyse des conséquences de l'ajustement. Si la stabilisation de l'économie bolivienne a été un succès, l'ajustement n'a pas pu remédier à la pauvreté dans le pays.

- Christian Suter, Weltwirtschaftliche Verflechtung. Regimewandel und Demokratisierung in Lateinamerika.

Le cas du Costa Rica montre qu'il est possible qu'un ajustement structurel réussisse économiquement sans une détérioration de la situation des droits de l'Homme.

- Gilbert Blardone/Lothar Caviezel, Stratégies de développement et ajustements structurels - Etude de l'appui de la Suisse aux programmes d'ajustement de deux de ses partenaires africains.

Analyse des conséquences économiques et sociales des programmes d'ajustement structurel à Madagascar et en Tanzanie. 
- Martin Hellwig, La problématique de l'endettement international - Causes, tendances et solutions possibles du point de vue de la Suisse.

- Othmart Schwank/Dieter Zürcher, Stadt-Land Beziehungen - Eine Herausforderung für die schweizerische Entwicklungszusammenarbeit.

Etude de cas du Népal. Cinq aspects des relations ville-campagne sont particulièrement importants pour le processus de développement: l'exode rural, le manque de décentralisation des décisions, l'inefficience des institutions étatiques en milieu rural, les déficiences en matière d'innovation ainsi que l'externalisation des coûts résultant de l'urbanisation.

- Jean-Pierre Jacob/François Margot/Paul Sauvin/Peter Uvin, La contribution des acteurs publics et privés au développement rural local des pays du Tiers Monde (Sénégal, Burkina-Faso, Burundi, Bolivie).

Rôle des autorités gouvernementales et administratives locales dans le processus de développement.

• Markus Wyss/Bruno Messerli/Thomas Straubhaar, Förderung einer ökologisch verträglichen wirtschaftlichen Zusammenarbeit mit weniger entwickelten Ländern. Et: Ernst Brugger/Martin Maurer, Fördern multinationale Firmen das Ökologie-Know-how in Entwicklungsländern?

La législation en matière d'environnement moins restrictive dans les pays en développement n'est pas une motivation suffisante pour inciter les entreprises multinationales à transférer une partie de leur production dans ces pays.

• Richard Gerster, Investir dans les intérêts communs.

La recherche examine les réformes nécessaires de la coopération au développement. L'auteur préconise notamment la conclusion d'accords de coopération symétriques à long terme entre la Suisse et les pays sur lesquels elle concentre les efforts de coopération.

Source: texte de l'encadré cité de l'ouvrage: Rolf Kappel/Olivier Landmann, La Suisse dans un monde en mutation - Economie extérieure et politique du développement: défis et perspectives, Fribourg, Editions Universitaires Fribourg, 1997. Dans les pages 437 à 453 se trouvent la liste des projets du PNR 28 et la liste des publications.

Rolf Kappel/Olivier Landmann, La Suisse dans un monde en mutation - Economie extérieure et politique du développement: défis et perspectives, Fribourg, Editions Universitaires Fribourg, 1997, 456 p.

Fonds national de recherche scientifique, dossier de presse, Berne, 26 août 1997, 456 pages.

Neue Zürcher Zeitung, 26.-27. September 1997 «Mehr Symmetrie zwischen Nord und Süd - Vom Forschungsprogramm zur Strukturanpassung». 


\section{3. ÉCHANGES CULTURELS SUD-NORD}

\section{$\square$ Importation des films de pays en développement}

Certains pays dominent nettement le marché cinématographique en Suisse et la place laissée aux films de pays en développement est très faible. Le tableau $\mathrm{n}^{\circ} 18$ ci-dessous montre le nombre de spectateurs par pays de provenance des films. La statistique est basée sur les données fournies par la majorité des cinémas commerciaux de Suisse. Ne sont par contre pas compris dans ces chiffres les spectateurs de festivals de films ou de projections de ciné-clubs. Les 1400 films environ projetés en 1996 ont attiré plus de 16,8 millions de spectateurs. La part du marché des films des Etats-Unis est en régression ces dernières années. La part des spectateurs ayant vu des films de ce pays dépassait les $80 \%$ au début des années 90 . Cette part était de $75 \%$ en 1994 et tourne autour des deux tiers en 1995 et 1996. La part des films européens est en revanche plus élevée ces dernières années, avec une part importante pour le cinéma français et britannique en 1996. La part des films de pays en développement est très faible $(1,1 \%$ du nombre total des spectateurs en 1996), alors que certains pays en développement produisent pourtant beaucoup de films.

\section{Tableau $\mathrm{n}^{\circ} 18$}

Nombre de spectateurs dans les cinémas selon le pays de provenance des films (année 1996)

\begin{tabular}{lrrr}
\hline $\begin{array}{l}\text { Pays de provenance } \\
\text { des films }\end{array}$ & $\begin{array}{r}\text { Nombre } \\
\text { de spectateurs }\end{array}$ & $\begin{array}{r}\text { En \% } \\
\text { du total }\end{array}$ & $\begin{array}{r}\text { Nombre } \\
\text { de films }\end{array}$ \\
\hline Etats-Unis & 10534245 & 62.6 & 571 \\
\hline France & 1887261 & 11.2 & 217 \\
\hline Royaume-Uni & 982715 & 5.8 & 95 \\
\hline Italie & 960306 & 5.7 & 46 \\
\hline Suisse & 707081 & 4.2 & $\mathbf{1 5 3}$ \\
\hline Allemagne & 570130 & 3.4 & 117 \\
\hline Europe centrale et orientale & 94670 & 0.6 & 31 \\
\hline Autres pays industrialisés & 902278 & 5.4 & 107 \\
\hline Pays en développement & 189438 & 1.1 & 62 \\
\hline \multicolumn{1}{c}{ dont: Amérique latine } & 110415 & 0.7 & 13 \\
\hline \multicolumn{1}{c}{ Asie } & 41099 & 0.2 & 29 \\
\hline \multicolumn{1}{c}{ Afrique } & 24625 & 0.1 & 13 \\
\hline Total & $\mathbf{1 6 8 2 8 1 2 4}$ & $\mathbf{1 0 0 . 0}$ & $\mathbf{1 3 9 9}$ \\
\hline
\end{tabular}

Source: calculé à partir des données statistiques in Procinéma, Association suisse des exploitants et distributeurs de films, Statistiques 1996, Berne, 1997.

La grande partie des entrées de cinéma se concentrent sur un nombre très restreint de films. Les dix films qui ont eu le plus de succès en 1996 ont attiré près du quart de tout le public de l'année. Deux films de pays en développement seulement figuraient parmi la liste des 100 plus grands succès à l'écran: 
Guantanamera de Thomas Gutierrez Alea (Cuba, au $71^{\mathrm{e}}$ rang, avec plus de 55'000 entrées) et No te mueras sin decirme adonde vas (Argentine, plus de 38'000 entrées). En 1995, on trouvait un autre film argentin parmi les plus grands succès à l'écran, Sur (plus de 60'000 en 1995). Le film cubain Fresa y chocolate s'est trouvé deux années de suite parmi les 100 films à succès (plus de 90'000 spectateurs en 1994 et 47'000 en 1995).

\section{$\square$ Promotion en Suisse des films de pays en développement}

Plusieurs initiatives ont été prises depuis le début des années 80 pour favoriser la production et la diffusion de films d'autres continents. Le Festival de films de Fribourg, créé au début des années 80, a présenté en mars 1997 plus de 70 films. D'autres festivals ou cycles de films permettent au public de voir des images d'autres continents: festival Cinemafrica tous les deux ans en novembre à Zurich, Festival de films de Locarno. Les journées de films et de vidéos Nord/Sud organisées par les œuvres d'entraide des Eglises permettent de présenter une sélection de films en distribution pour les écoles" (Filme für eine Welt). Les Rencontres médias Nord-Sud présentent chaque année en mai des documentaires de chaînes de télévision ou des documentaires de producteurs indépendants. La fondation Trigon-film est une maison de distribution diffusant uniquement des films de réalisateurs d'Afrique, d'Asie ou d'Amérique latine. Plus d'une soixantaine de films ont ainsi été diffusés depuis une dizaine d'années.

La Direction du développement et de la coopération (DDC) permet aussi d'aider l'expression artistique et de favoriser la rencontre entre les cultures en soutenant la production et la diffusion de films de pays du Sud ou de l'Europe orientale. Pour la DDC, le cinéma est un moyen de sensibilisation et d'information qui permet de mieux connaître et comprendre «l'autre», de mieux nous approcher de certaines réalités du monde, et donc aussi de nous ouvrir à la solidarité. Le Sud peut aussi par ce biais nous apporter quelque chose, et non pas être uniquement receveur d'aide ${ }^{10}$. La fondation Montecinemaverità, cofinancée par la DDC et Benetton-Suisse, a permis depuis octobre 1992 d'appuyer le financement de la production de 34 films.

La contribution annuelle de la DDC pour le cinéma des pays du Sud ou de l'Europe orientale s'est élevée à 830'000 francs en 1997, dont 200'000 francs pour la diffusion de films du Sud en Suisse (Trigon-film), 200'000 francs pour l'organisation de festivals ou cycles de films et 330'000 francs pour la fondation Montecinemaverità en 1997 (qui s'ajoutent aux 310'000 francs versés par Benetton). Parmi les films récents soutenus par la fondation, on peut relever $B a b$ El Oued City (Algérie), L'arche du désert de l'Algérien Mohamed Chouikh, Dance of the Wind (Inde). La DDC apporte son soutien financier à Trigon-film, le Festival de films de Fribourg, Cinemafrica, Black Movie, les Rencontres médias Nord-Sud et les Weltfilmtage à Thusis. Cent mille francs ont en outre per-

9. Des films de fiction ou des documentaires de pays en développement sont disponibles pour la distribution dans les écoles (ou dans certains cas pour les ciné-clubs) aux adresses suivantes. La sélection des œuvres d'entraide des Eglises est disponible à Zoom-Filmverleih. Les films Trigon (et d'autres films) peuvent être commandés au FilmInstitut, tél. 031/301.01.16 (vidéos en vente à la DDC). Cinédia diffuse une série de longs et courts-métrages pour la distribution non commerciale et vend des vidéos: Cinédia, case postale 197, 1701 Fribourg, tél. 026/426.34.30

10. Interview de Marco Cameroni, du service d'information de la DDC, in La Regione Ticino, 14 agosto 1997. 
mis de financer en 1997 la production de films documentaires (budget de $125^{\prime} 000$ francs en 1998). En dehors des 830 '000 francs d'encouragement au cinéma, la DDC a aussi versé $680^{\prime} 000$ francs pour la promotion de rencontres culturelles (musique, théâtre, publications), dont le soutien à Culture et Développement.

\section{$\square$ Arts et cultures des pays du Sud}

Une trentaine d'organisations suisses ont à des degrés divers pour objectif de promouvoir l'art des pays en développement (musique, films, théâtre, littérature, photographie, expositions, art contemporain, promotion de tournées d'artistes, festivals de films ou de musique). Plusieurs organisations privées d'entraide consacrent une partie de leurs activités à mieux faire connaître la culture d'autres pays ${ }^{11}$. La fondation Pro Helvetia encourage des activités culturelles susceptibles de contribuer au dialogue entre la Suisse et les pays en développement, par le biais du service Echanges culturels Sud-Nord (budget annuel de 430'000 francs environ). Culture et Développement est un réseau regroupant des œuvres d'entraide, qui encourage et coordonne en Suisse la présentation de la culture des autres continents.

\section{SOURCES}

Procinéma, Association suisse des exploitants et distributeurs de films, Statistiques 1996, Berne, 1997. Basler Zeitung, 8.-10. August 1997 (fondation Montecinemaverità).

La Regione Ticino, 14 agosto 1997 «Marco Cameroni illustra il ruolo della DSC all'interno di Montecinemaverità».

11. Parmi les organisations engagées dans la promotion d'activités culturelles, on peut citer les institutions suivantes: Action de Carême, Caritas, Déclaration de Berne, EPER, Helvetas, organisations d'aide aux réfugiés, Pain pour le prochain, Swissaid, Terre des hommes Suisse, UNICEF. 